\title{
Consolidation of Associative and Item Memory Is Related to Post-Encoding Functional Connectivity between the Ventral Tegmental Area and Different Medial Temporal Lobe Subregions during an Unrelated Task
}

\author{
Alexa Tompary, ${ }^{1 *}$ Katherine Duncan, ${ }^{3 *}$ and Lila Davachi ${ }^{1,2}$ \\ ${ }^{1}$ Department of Psychology and ${ }^{2}$ Center for Neural Science, New York University, New York, New York 10003 and ${ }^{3}$ Department of Psychology, Columbia \\ University, New York, New York 10027
}

\begin{abstract}
It is well established that the hippocampus and perirhinal cortex $(\operatorname{PrC})$ encode associative and item representations, respectively. However, less is known about how item and associative memories are consolidated. We used high-resolution fMRI in humans to measure how functional connectivity between these distinct medial temporal lobe regions with the ventral tegmental area (VTA) after a paired associate encoding task is related to both immediate and $24 \mathrm{~h}$ item and associative memory performance. We found that the strength of post-encoding functional connectivity between the VTA and CA1 selectively correlated with long-term associative memory, despite subjects actively engaging in an unrelated task during this period. Conversely, VTA-PrC functional connectivity during the same period correlated with long-term item memory. Critically, connectivity between VTA and these MTL regions were only related to memory tested at a $24 \mathrm{~h}$ delay, implicating midbrain connectivity in the consolidation of distinct forms of memory.
\end{abstract}

Key words: consolidation; functional connectivity; high-resolution fMRI; medial temporal lobe; VTA

\section{Introduction}

Some elements of our rich experiences persist as long-term memories, whereas others are forgotten. It is well established that regions within the medial temporal lobe (MTL) support the encoding of distinct elements of our experiences. Specifically, hippocampal activation has been linked with successful associative encoding, whereas engagement of the underlying perirhinal cortex $(\mathrm{PrC})$ is related to item memory (Brown and Aggleton, 2001; Davachi et al., 2003; Ranganath et al., 2004; Davachi, 2006). However, the longevity of memories is not only dependent on encoding processes but also depends on post-encoding consolidation processes.

In rodents, post-encoding patterns of hippocampal neural firing have been shown to "replay" during sleep and rest (Wilson and McNaughton, 1994; Foster and Wilson, 2006). Similarly, in humans, memory performance has been shown to correlate with experience-dependent hippocampal-cortical functional connectivity (Tambini et al., 2010), as well as persistence of hippocampal and entorhinal multivoxel patterns into rest and active tasks, re-

\footnotetext{
Received Nov. 25, 2014; revised Feb. 25, 2015; accepted March 25, 2015.

Author contributions: K.D. and L.D. designed research; K.D. performed research; A.T. and K.D. analyzed data; A.T., K.D., and L.D. wrote the paper.

This work was supported by National Institute of Mental Health Grant MH074692 (L.D.).

*A.T. and K.D. contributed equally to this work.

The authors declare no competing financial interests.

Correspondence should be addressed to Lila Davachi, Department of Psychology, New York University, 6 Washington Place, 8th floor, New York, NY 10003. E-mail: lila.davachi@nyu.edu.

DOI:10.1523/JNEUROSCI.4816-14.2015

Copyright $\odot 2015$ the authors $\quad 0270-6474 / 15 / 357326-06 \$ 15.00 / 0$
}

spectively (Staresina et al., 2013; Tambini and Davachi, 2013). However, it remains unclear how post-encoding processes relate to item and associative memory consolidation.

One consolidation mechanism that may be common to the hippocampus and $\mathrm{PrC}$ is dopaminergic facilitation of long-term potentiation (LTP). This is motivated by three key points: (1) dopamine antagonists block long-term memory when injected into hippocampal areas CA1 and PrC (O'Carroll et al., 2006; Bethus et al., 2010; Balderas et al., 2013); (2) dopaminergic manipulations influence the late, but not early, phase of LTP (Frey et al., 1990; Huang and Kandel, 1995; Li et al., 2003) and the expression of memories tested at long $(>6 \mathrm{~h})$, but not short, delays (O'Carroll et al., 2006; Bethus et al., 2010; Balderas et al., 2013), suggesting that dopamine regulates the stabilization rather than initial formation of memory traces; and (3) memory performance can be modulated by post-encoding injections of dopamine agonists (Packard and White, 1991) and post-encoding exposure to novelty via dopamine-dependent mechanisms (Moncada and Viola, 2007; Wang et al., 2010). Together, these findings suggest that memory consolidation in both the hippocampus and $\mathrm{PrC}$ may be influenced by post-encoding interactions with their primary dopamine input structure, the ventral tegmental area (VTA).

We tested this hypothesis in the current high-resolution fMRI experiment. Participants alternated between encoding and retrieving object pairs, with an active post-encoding delay period interleaved between each encoding-retrieval block. Twenty-four hours later, participants' associative and item memory were 
A

Day 1 (scanned)
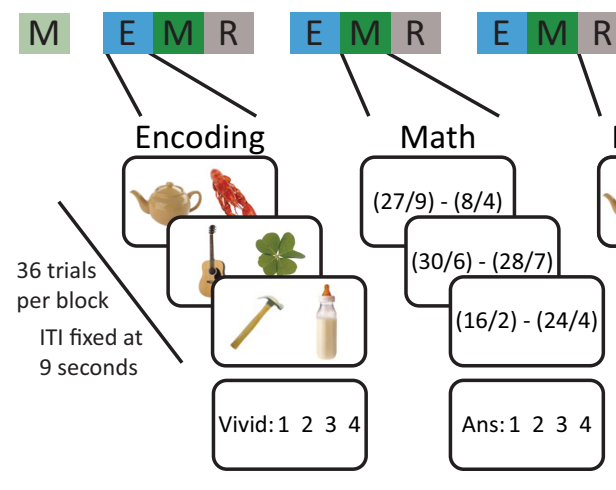

B
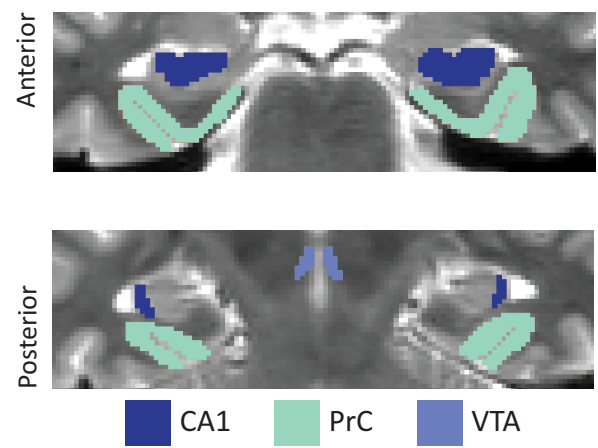

Day 2
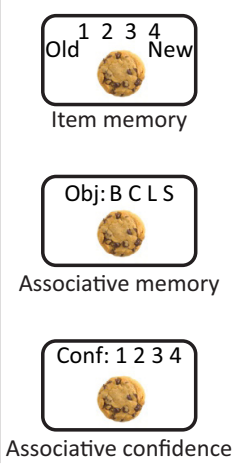

C

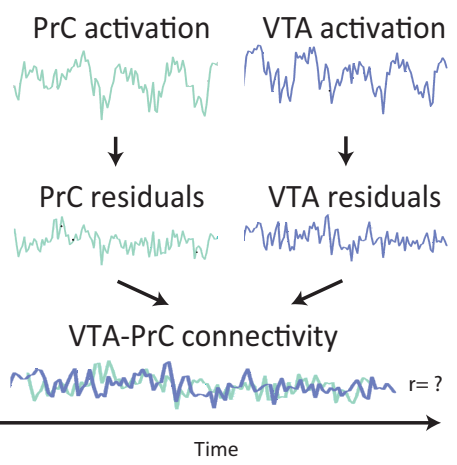

Figure 1. A, Experiment design. On day 1, participants completed blocks of encoding (E), math (M), and retrieval (R), after one block of baseline math. During encoding, participants imagined a unique cue interacting with one of four repeating associates. During math blocks, participants solved math problems. During retrieval, participants viewed a cue and were asked to retrieve its associated object. On day 2, participants were tested for associative and item memory. $\boldsymbol{B}$, ROl segmentation. One participant's T2-weighted coronal slice displaying ROIs. C, Background connectivity. Preprocessed volumes were entered into a voxelwise GLM capturing nuisance signals and trial-evoked activity. The residuals were bandpass filtered, and the mean signal across each ROI was extracted over the duration of each math and encoding block. ITI, Intertrial interval.

tested. Focusing our analyses on VTA functional connectivity with $\mathrm{CA} 1$ and $\mathrm{PrC}$, we predicted that changes in VTA functional connectivity during these periods would predict (1) long-term, but not short-term, memory and (2) associative and item memory, respectively.

\section{Materials and Methods}

Results from the encoding and retrieval scans have been published previously (Duncan et al., 2014). An expanded description of the behavioral task, scan sequences, and region of interest (ROI) tracing can be found there.

Participants. Seventeen students from New York University participated (eight females; mean age, 27.1 years; range, 22-35 years). Three participants with memory performance of $2.5 \mathrm{SD}$ below the mean, who reported falling asleep during the scanning session, were excluded. One additional participant was removed because of experimenter error. All participants were right-handed with normal or corrected vision. The Institutional Review Board at New York University approved all procedures.

Experiment design. The experiment consisted of two sessions separated by $24 \mathrm{~h}$. On the first day, participants were scanned while completing blocks of encoding, post-encoding math, and retrieval tasks, preceded by a block of pre-encoding baseline math (Fig. 1A). Each block lasted for 5.5 min, broken into 36 trials with identical trial onsets (every $9 \mathrm{~s}$ ), stimulus periods ( $3 \mathrm{~s})$, response windows ( $2 \mathrm{~s})$, and fixation periods (4s). In all tasks, participants were encouraged to opt out using the thumb button rather than guessing. On the second day, participants completed recognition and cued recall tests.
Encoding task. Pairs of objects were presented on each encoding trial. Participants were instructed to vividly imagine the two objects interacting and then rate the vividness of their imagined scenario on a scale of 1-4. Critically, the object on the left side was only presented once ("cue"), whereas the object on the right was one of four repeating "associates": (1) baby bottle; (2) clover; (3) lobster; or (4) scissors.

Baseline and post-encoding math task. Participants solved math problems that required the subtraction of two dividends. The solution to every problem was $1,2,3$, or 4 , corresponding to the four button-box keys. Critically, the first math block was completed before the first encoding block, allowing for an assessment of baseline functional connectivity.

Immediate retrieval task. After each postencoding math block, object associations from the immediately preceding encoding block were tested using cued recall. On each trial, participants were instructed to match a presented cue with the correct associate, using response options corresponding to the names of the four possible associates. A constant placeholder image appeared next to each cue.

Long-term memory test. Twenty-four hours later, participants completed a self-paced threestep memory task to separately assess item and associative memory with confidence. On each trial, participants were instructed to indicate whether the presented object was old or new using a scale that included confidence (definitely old, probably old, don't know, probably new, definitely new). If they selected an "old" response, they were then asked to match the object with the correct associate (B, C, L, S, don't know). Finally, they rated the confidence of this choice on a scale of $1-4$. These responses were divided into a highconfident (HC) bin, using responses 3 and 4 , and a low-confident (LC) bin, using responses 1 and 2 .

Behavioral measures. We computed three memory scores for each participant. First, we computed long-term associative memory using the day $2 \mathrm{HC}$ cued recall responses: (HC correct recall - HC incorrect recall/4)/ 144. The incorrect responses were divided by four to account for the larger proportion of incorrect response options presented on each trial (Duncan et al., 2014). For long-term item memory, we computed corrected recognition from day 2 , limited to $\mathrm{HC}$ responses. Importantly, we only included hits with LC or incorrect cued recall to dissociate item memory from associative memory performance: (HC hits with LC or incorrect source/all old items with LC or incorrect source) - (HC false alarm/72). Finally, we computed an immediate memory score using day 1 cued recall: (source correct - source incorrect/4)/144.

fMRI parameters. All scanning was performed using a 3T Siemens Allegra MRI system with a whole-head coil. Projected visual stimuli were viewed through a mirror attached to the head coil. Functional data were collected using a high-resolution echo-planar pulse (echo-planar imaging), with oblique coronal slices aligned perpendicular to the long axis of the hippocampus [1500 ms repetition time (TR); $22 \mathrm{~ms}$ echo time (TE); field of view, $192 \times 96 ; 21$ slices; $1.5 \times 1.5 \times 3 \mathrm{~mm}$ voxels; $77^{\circ} \mathrm{flip}$ angle]. We also collected a whole-brain T1-weighted MPRAGE $(1 \times 1 \times 1 \mathrm{~mm}$ voxels, 176 sagittal slices), a field-map sequence, and a T2-weighted image $(5100 \mathrm{~ms}$ TR, $88 \mathrm{~ms}$ TE, $0.898 \times 0.898 \times 1.5 \mathrm{~mm}$ voxels $)$ in the same plane as the functional volumes.

ROI segmentation. We manually drew ROIs using each participant's high-resolution MPRAGE and T2 scans (Fig. 1B). Hippocampal area CA1 was isolated by matching each slice to plates from an atlas (Duvernoy, 2005) using a procedure outlined by Kirwan et al. (2007). PrC and 
Math connectivity and associative memory

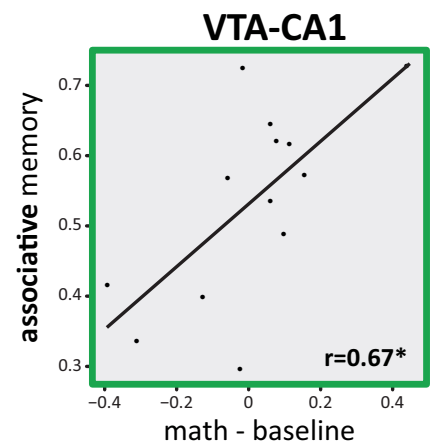

correlation $(\mathrm{z})$

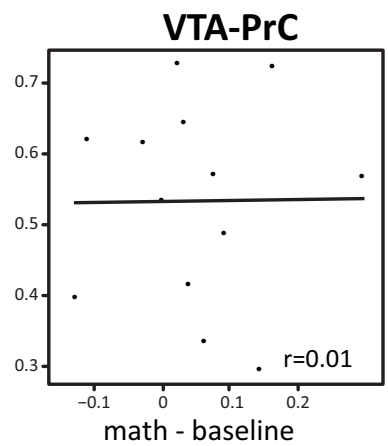

correlation (z)

\section{Correlation with item memory} VTA-CA1
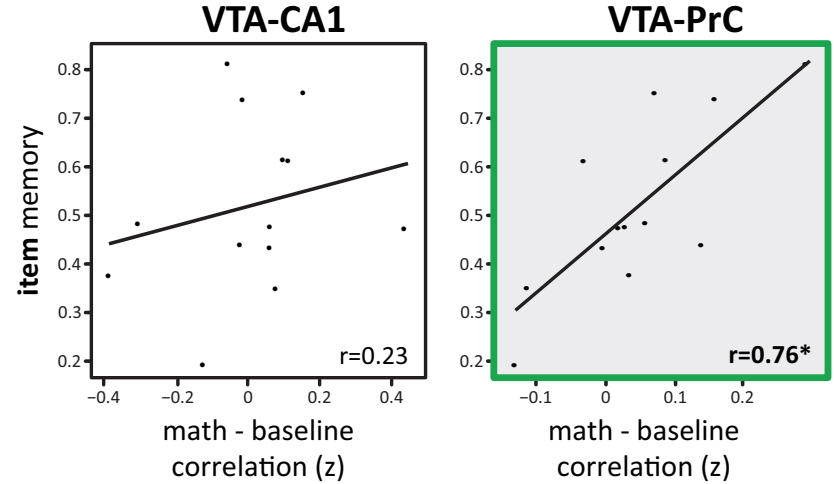

Figure 2. Correlations with VTA connectivity. Participants with a larger increase in VTA-CA1 and VTA-PrC connectivity during post-encoding math relative to baseline math have greater associative memory and item memory, respectively. ${ }^{*} p<0.05$.

VTA were demarcated using guidelines developed by Insausti et al. (1998) and D'Ardenne et al. (2008), respectively. These ROIs, in addition to hippocampal subfields CA3/dentate gyrus and subiculum, as well as entorhinal and parahippocampal cortices, were originally segmented for use by Duncan et al. (2014). The ROIs were resampled to functional resolution, aligned with the functional volumes, and restricted to voxels with sufficient signal-to-noise to exclude areas that suffered from dropout, using the 3dAutomask function (AFNI: http://afni.nimh.nih. gov/afni) and visual inspection.

fMRI preprocessing. The data were preprocessed using custom scripts combining FSL (FMRIB Software Library) command line tools (http://www.fmrib.ox.ac.uk/fsl) and AFNI. After discarding the first 11 volumes, runs were slice-time corrected, corrected for motion, and registered to anatomical images using targets produced by the field-map sequences.

Background connectivity. Functional connectivity was measured using the correlation in low-frequency fluctuations during the encoding and math tasks. Importantly, this "background connectivity" is unrelated to the trial-evoked responses (Fig. 1C) and has been used previously to identify mechanisms of sustained attention (Al-Aidroos et al., 2012; Norman-Haignere et al., 2012) and distinguish between encoding and retrieval pathways in the hippocampus (Duncan et al., 2014).

We first removed trial-evoked and nuisance signals using a voxelwise GLM. Each trial was modeled as a 3 s boxcar convolved with the HRF and its temporal and dispersion derivatives with trials binned according to math accuracy and subsequent memory performance (Duncan et al., 2014). We included the six motion parameters and the mean activity in the ventricles and white matter tracts as separate regressors. We then bandpass filtered the residuals of this model, leaving only signal between 0.01 and $0.1 \mathrm{~Hz}$ (Cordes et al., 2001). Critically, this band is outside the task frequency $(0.11 \mathrm{~Hz})$, filtering out responses that were consistently elicited by math trials.
Table 1. Statistical tests of the correlation of functional connectivity between VTA-CA1 and VTA-PrC during post-encoding math with long-term associative and item memory

\begin{tabular}{|c|c|c|c|c|}
\hline \multirow[b]{2}{*}{$\begin{array}{l}\text { Partial correlation: } \\
\text { immediate memory }\end{array}$} & \multicolumn{2}{|c|}{ Long-term associative memory } & \multicolumn{2}{|c|}{ Long-term item memory } \\
\hline & $r$ & $p$ & $r$ & $p$ \\
\hline VTA-CA1 & 0.61 & 0.04 & 0.15 & 0.65 \\
\hline VTA-PrC & -0.28 & 0.38 & 0.59 & 0.04 \\
\hline $\begin{array}{l}\text { Partial correlation: } \\
\text { encoding connectivity }\end{array}$ & $r$ & $p$ & $r$ & $p$ \\
\hline VTA-CA1 & 0.76 & 0.004 & 0.32 & 0.31 \\
\hline VTA-PrC & -0.24 & 0.45 & 0.28 & 0.38 \\
\hline Permutation test & $r$ & $p$ & $r$ & $p$ \\
\hline VTA-CA1 & 0.67 & 0.005 & 0.32 & 0.14 \\
\hline VTA-PrC & 0.01 & 0.50 & 0.62 & 0.01 \\
\hline Robust regression & $\beta$ & $p$ & $\beta$ & $p$ \\
\hline VTA-CA1 & 1.00 & 0.02 & 0.47 & 0.19 \\
\hline VTA-PrC & -0.06 & 0.83 & 0.40 & 0.04 \\
\hline Cross-validation & \% Error & $p$ & \% Error & $p$ \\
\hline VTA-CA1 & 8.8 & $<0.001$ & 15.0 & 0.57 \\
\hline VTA-PrC & 13.3 & 0.62 & 12.6 & 0.06 \\
\hline
\end{tabular}

We controlled for immediate memory and encoding connectivity using partial correlations. To confirm that these findings were reliable, we used a permutation test, robust regression, and a cross-validation procedure. Bold $p$ values indicate significance at $p<0.05$.

For each ROI, we extracted mean signal from the filtered residuals over all volumes corresponding to the encoding and math tasks. Time series within a task were then concatenated across runs and correlated across ROIs. The resulting correlations were Fisher transformed.

Correlations between math task connectivity and memory. The three behavioral memory scores were correlated with participants' background connectivity during post-encoding math tasks. To ensure that these correlations did not reflect individual differences in participants' general connectivity, we first subtracted out functional connectivity during the baseline math task.

To assess the reliability and specificity of identified relationships between functional connectivity and behavior, we used partial correlations, robust regression, and permutation tests using a distribution of memory correlations created by shuffling memory performance across participants 10,000 times. We also used cross-validation to predict each participant's long-term item and associative memory (Duncan et al., 2014). First, the relationship between functional connectivity and memory was estimated independently using the remaining participants. We then used these models to predict the left-out participant's memory and quantified the prediction accuracy as the absolute value of the difference between the predicted and actual memory. These predictions were compared against a null distribution created from repeating the above regressions 10,000 times with memory scores shuffled across participants.

Temporal relationship between math connectivity and memory. Although math blocks always directly followed encoding blocks, the repeating cycles of encoding, math, and retrieval tasks in this design resulted in math blocks that both preceded and followed different encoding task blocks. This allowed us to assess whether later memory was more related to functional connectivity following encoding relative to functional connectivity preceding encoding. We correlated associative and item memory for each of the four blocks with functional connectivity during each of the four math blocks across participants. We then binned the correlation values by whether the math block functional connectivity preceded or followed the encoding block from which the memory scores were computed. To assess pre-encoding versus post-encoding differences, we generated a null distribution by repeating the above analysis 10,000 times but randomly selected the behavioral run entered into each correlation. To test how pre-encoding versus post-encoding functional connectivity related to associative and item memory, we repeated the above analysis 
VTA-CA1 math connectivity

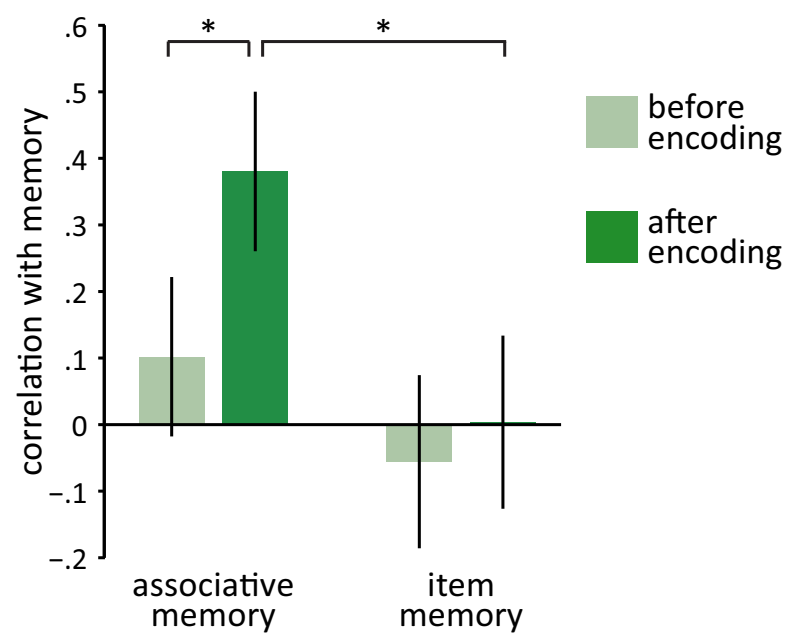

VTA-PrC math connectivity

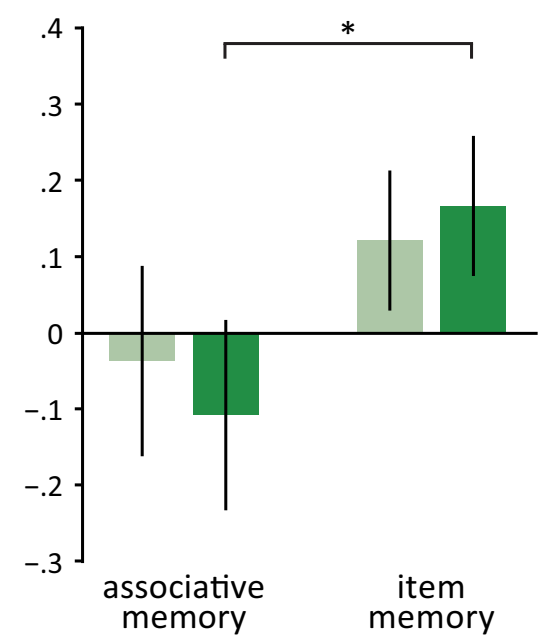

Figure 3. Correlation between math connectivity and memory as a function of pre-encoding or post-encoding. VTA-CA1 connectivity was more correlated with associative memory and VTA-PrC connectivity with item memory when restricted to post-encoding math. Error bars indicate the standard error of the mean. ${ }^{*} p<0.05$, one tailed.

and randomly selected item or associative memory performance over 10,000 iterations.

Relationship between encoding task and math task connectivity. We also assessed whether changes in functional connectivity during the postencoding math tasks were related to changes evoked by the preceding encoding tasks. Specifically, we correlated functional connectivity during these tasks across subjects and also used partial correlations to determine whether the relationship between memory and math task connectivity was independent of the relationship between memory and encoding task connectivity.

\section{Results}

Behavior

Immediate cued recall performance was reliably above chance (mean $\pm \mathrm{SD}, 0.79 \pm 0.15 \%$ correct, $t_{(15)}=14.47, p<10^{-11}$ ), and performance on the math task was consistently high $(0.76 \pm$ $0.13 \%$ correct $)$. We found a marginal improvement in math performance between the baseline and post-encoding math blocks $\left(t_{(15)}=1.92, p=0.073\right)$. On day 2, participants identified old and new objects with $89.9 \pm 10.6 \%$ accuracy (mean \pm SD) and matched old objects to their encoded associates reliably above chance $\left(65.2 \pm 20.3 \%, t_{(15)}=7.91, p<10^{-7}\right)$.

\section{Memory correlations with VTA-MTL connectivity}

We reported previously that VTA-CA1 functional connectivity during encoding correlated with long-term associative memory (Duncan et al., 2014). Here, we assessed whether functional connectivity during the subsequent post-encoding periods also correlated with long-term memory. Although there was no overall shift in VTA-CA1 functional connectivity with encoding experience, VTA-CA1 connectivity during post-encoding math correlated positively with long-term associative memory across participants (math: $r_{(11)}=0.67, p<0.05$ ). Critically, VTA-CA1 functional connectivity was not significantly correlated with later item memory $\left(r_{(11)}=0.23, p=0.45\right)$. In contrast, VTA-PrC functional connectivity correlated positively with long-term item memory $\left(r_{(11)}=0.76, p<0.005\right)$ but not long-term associative memory performance $\left(r_{(11)}=0.01, p=0.97\right.$; Fig. 2$)$. To confirm that these correlations were robust and generalizable, we replicated these effects using robust regression and cross-validation (Table 1).

\section{Specificity of VTA connectivity and forms of memory}

To measure the specificity of these relationships, we used a permutation test by shuffling associative and item memory scores across participants. We found that post-encoding VTA-PrC functional connectivity was more correlated with item memory than associative memory (one-tailed $p=0.004$ ), whereas VTACA1 functional connectivity was marginally more correlated with associative memory than item memory (one-tailed $p=0.09$ ). Importantly, the interaction between VTA-MTL functional connectivity and the form of memory [(VTA-CA1 correlation with associative - VTA-CA1 correlation with item) - (VTA-PrC correlation with associative - VTA-PrC correlation with item) ] was significant (one-tailed $p=0.006$ ), suggesting that VTA-MTL interactions after encoding differentially relate to associative and item memory as a function of MTL region.

\section{VTA-MTL connectivity and immediate memory}

Critically, functional connectivity during post-encoding math blocks did not reliably predict immediate memory performance $\left(\right.$ VTA-CA1: $r_{(11)}=0.41, p=0.17$; VTA-PrC: $r_{(11)}=0.26, p=$ $0.40)$. Furthermore, when controlling for immediate memory using a partial correlation, VTA-PrC and VTA-CA1 functional connectivity during math still positively correlated with longterm item and associative memory, respectively (Table 1). Thus, post-encoding functional connectivity between VTA and these MTL regions is related to long-term, but not immediate, memory performance.

\section{Temporal relationship between math connectivity and memory}

We next asked whether functional connectivity during math blocks after encoding (post-encoding) was more related to later memory than math blocks preceding it (pre-encoding) by correlating memory with functional connectivity on a run-by-run basis. We found that post-encoding VTA-CA1 functional connectivity was more correlated with long-term associative memory than pre-encoding connectivity (one-tailed $p=0.02$; Figure 3). However, post-encoding VTA-PrC math functional connectivity was not significantly more correlated with long-term item 
memory than pre-encoding connectivity (one-tailed $p=0.20$ ). Importantly, these analyses also confirmed that VTA-CA1 postencoding math functional connectivity was more correlated with later associative relative to item memory (one-tailed $p=0.01$ ), whereas VTA-PrC post-encoding math functional connectivity was more correlated with later item than associative memory (one-tailed $p=0.02$ ). The same relationships were not found between pre-encoding connectivity and later memory (all $p$ values $>0.18)$. Together, these results suggest that the consolidation of memories supported by distinct MTL regions is related specifically to post-encoding functional connectivity with VTA.

\section{Relationship between encoding task and post-encoding math connectivity}

In this same dataset, we reported previously that VTA-CA1 functional connectivity during the encoding task predicted long-term associative memory (Duncan et al., 2014), complementing the current findings that VTA-MTL connectivity during the postencoding math task predicts long-term associative and item memory. We next investigated whether processes occurring during these two tasks independently relate to long-term memory performance. First, we found that encoding and math task connectivity were not correlated significantly across subjects (VTACA1: $r_{(11)}=0.19, p=0.53$; VTA-PrC: $\left.r_{(11)}=0.09, p=0.77\right)$. Furthermore, when accounting for VTA-CA1 functional connectivity during the encoding task using a partial correlation, we found that VTA-CA1 post-encoding math connectivity still correlated with long-term associative memory $\left(r_{(11)}=0.76, p=\right.$ $0.004)$ but not long-term item memory $\left(r_{(11)}=0.32, p=0.31\right)$. Together, this suggests that connectivity during the encoding and post-encoding tasks reflect distinct processes that relate to longterm associative memory. Interestingly, when using a partial correlation to account for VTA-PrC encoding connectivity, VTA-PrC post-encoding connectivity did not relate to later item memory $\left(r_{(11)}=0.28, p=0.38\right)$. This may hint at a separate mechanism underlying the consolidation of long-term item memory.

\section{Discussion}

We found that post-encoding changes in the functional connectivity between the VTA and anatomically connected MTL regions relate to subsequent long-term memory across individuals in specific ways: VTA-CA1 connectivity is related to later associative memory, VTA-PrC connectivity is related to later item memory, and VTA-MTL connectivity is related to memory after a long, but not short, delay. Together, these findings suggest that, after encoding, interactions between the VTA and the MTL facilitate the stabilization of specific ongoing processes supported by that distinct MTL region.

Previous event-related functional imaging work has shown that hippocampal blood-oxygen-level dependent (BOLD) activation during encoding trials is related to later associative memory, whereas BOLD activation in the $\mathrm{PrC}$ is related to later item memory (Davachi et al., 2003; Ranganath et al., 2004; Uncapher et al., 2006; Staresina and Davachi, 2008; for a review, see Davachi, 2006). Here we show that post-encoding functional connectivity between these MTL regions and the VTA is also selectively related to long-term associative and item memory. These results additionally extend previous fMRI studies that identified evidence for midbrain contributions to memory (Wittmann et al., 2005; Adcock et al., 2006; Shohamy and Wagner, 2008; Duncan et al., 2014; Murty and Adcock, 2014), by dissociating how func- tional connectivity between the VTA and distinct MTL regions support the formation of associative and item memories.

Interestingly, there is some evidence for a similar dissociation in rodent pharmacological research, such that manipulating dopamine in the hippocampus influences spatial and relational memory, whereas modulation of dopamine in the PrC influences object recognition memory (Packard and White, 1991; O'Carroll et al., 2006; Bethus et al., 2010; Balderas et al., 2013). Here we demonstrated that temporal correlations between the BOLD responses in MTL regions and their primary source of dopamine, the VTA (Akil and Lewis, 1993; Gasbarri et al., 1994), were related to memory consolidation. It is important to point out that the VTA BOLD signal reflects an amalgam of metabolically demanding processing, including the release of dopamine, as well as the firing of GABA and glutaminergic neurons (Grace et al., 2007); thus, we cannot make strong conclusions about dopamine using BOLD fMRI measures. However, the relationship between VTA functional connectivity and subsequent memory reported here is broadly consistent with a dopaminergic mechanism. Specifically, there is considerable evidence that dopamine supports the formation of long-lasting memories (Shohamy and Adcock, 2010). Consistent with this mechanism, functional connectivity between the VTA and the MTL was related to long-term, but not immediate, memory performance. Moreover, post-encoding dopaminergic manipulations have been shown to influence consolidation (Packard and White, 1991; Moncada and Viola, 2007; Wang et al., 2010). Similarly, we show that the dissociable relationship between memory form and VTA functional connectivity is limited to periods following encoding. Although future pharmacological work is needed to characterize the mechanism by which these interactions support memory consolidation, this result opens up promising questions about the timescale over which dopamine can modulate memory.

Importantly, past work has shown that experience-dependent changes in functional connectivity measured during rest are related to later memory (Tambini et al., 2010; Tambini and Davachi, 2013). By measuring functional connectivity during a challenging math task, we demonstrated that ongoing consolidation processes might also occur during active post-encoding periods. Two studies have found evidence for post-encoding processes during unrelated tasks (Peigneux et al., 2006; Staresina et al., 2013). Because these studies only examined memory after a short delay, it is unclear whether cortical reactivation in these studies relates specifically to memory consolidation. In contrast, we found that post-encoding VTA functional connectivity was related specifically to long-term memory, a pattern that strongly implicates consolidation processes.

In conclusion, we have demonstrated that MTL-VTA functional connectivity after encoding relates to long-term memory in a manner that is consistent with a large body of anatomical, physiological, and imaging work linking the hippocampus to associative memory and the PrC to item memory. Together, this experiment extends this body of research by identifying interactions between these distinct MTL regions and neuromodulatory centers that may support the consolidation of distinct elements of memory.

\section{References}

Adcock RA, Thangavel A, Whitfield-Gabrieli S, Knutson B, Gabrieli JDE (2006) Reward-motivated learning: mesolimbic activation precedes memory formation. Neuron 50:507-517. CrossRef Medline

Akil M, Lewis DA (1993) The dopaminergic innervation of monkey entorhinal cortex. Cereb Cortex 3:533-550. CrossRef Medline

Al-Aidroos N, Said CP, Turk-Browne NB (2012) Top-down attention 
switches coupling between low-level and high-level areas of human visual cortex. Proc Natl Acad Sci U S A 109:14675-14680. CrossRef Medline

Balderas I, Moreno-Castilla P, Bermudez-Rattoni F (2013) Dopamine D1 receptor activity modulates object recognition memory consolidation in the perirhinal cortex but not in the hippocampus. Hippocampus 23:873878. CrossRef Medline

Bethus I, Tse D, Morris RGM (2010) Dopamine and memory: modulation of the persistence of memory for novel hippocampal NMDA receptordependent paired associates. J Neurosci 30:1610-1618. CrossRef Medline

Brown MW, Aggleton JP (2001) Recognition memory: what are the roles of the perirhinal cortex and hippocampus? Nat Rev Neurosci 2:51-61. CrossRef Medline

Cordes D, Haughton VM, Arfanakis K, Carew JD, Turski PA, Moritz CH, Quigley MA, Meyerand ME (2001) Frequencies contributing to functional connectivity in the cerebral cortex in "resting-state" data. AJNR Am J Neuroradiol 22:1326-1333. Medline

D’Ardenne K, McClure SM, Nystrom LE, Cohen JD (2008) Bold responses reflecting dopaminergic signals in the human ventral tegmental area. Science 319:1264-1267. CrossRef Medline

Davachi L (2006) Item, context and relational episodic encoding in humans. Curr Opin Neurobiol 16:693-700. CrossRef Medline

Davachi L, Mitchell JP, Wagner AD (2003) Multiple routes to memory: distinct medial temporal lobe processes build item and source memories. Proc Natl Acad Sci U S A 100:2157-2162. CrossRef Medline

Duncan K, Tompary A, Davachi L (2014) Associative encoding and retrieval are predicted by functional connectivity in distinct hippocampal area CA1 pathways. J Neurosci 34:11188-11198. CrossRef Medline

Duvernoy HM (2005) The human hippocampus: functional anatomy, vascularization, and serial sections with MRI, Ed 3. Berlin: Springer.

Foster DJ, Wilson MA (2006) Reverse replay of behavioural sequences in hippocampal place cells during the awake state. Nature 440:680-683. CrossRef Medline

Frey U, Schroeder H, Matthies H (1990) Dopaminergic antagonists prevent long-term maintenance of posttetanic LTP in the CA1 region of rat hippocampal slices. Brain Res 522:69-75. CrossRef Medline

Gasbarri A, Packard MG, Campana E, Pacitti C (1994) Anterograde and retrograde tracing of projections from the ventral tegmental area to the hippocampal formation in the rat. Brain Res Bull 33:445-452. CrossRef Medline

Grace AA, Floresco SB, Goto Y, Lodge DJ (2007) Regulation of firing of dopaminergic neurons and control of goal-directed behaviors. Trends Neurosci 30:220-227. CrossRef Medline

Huang YY, Kandel ER (1995) D1/D5 receptor agonists induce a protein synthesis-dependent late potentiation in the CA1 region of the hippocampus. Proc Natl Acad Sci U S A 92:2446-2450. CrossRef Medline

Insausti R, Juottonen K, Soininen H, Insausti AM, Partanen K, Vainio P, Laakso MP, Pitkänen A (1998) MR volumetric analysis of the human entorhinal, perirhinal, and temporopolar cortices. AJNR Am J Neuroradiol 19:659-671. Medline

Kirwan CB, Jones CK, Miller MI, Stark CEL (2007) High-resolution fMRI investigation of the medial temporal lobe. Hum Brain Mapp 28:959-966. CrossRef Medline

Li S, Cullen WK, Anwyl R, Rowan MJ (2003) Dopamine-dependent facili- tation of LTP induction in hippocampal CA1 by exposure to spatial novelty. Nat Neurosci 6:526-531. CrossRef Medline

Moncada D, Viola H (2007) Induction of long-term memory by exposure to novelty requires protein synthesis: evidence for a behavioral tagging. J Neurosci 27:7476-7481. CrossRef Medline

Murty VP, Adcock RA (2014) Enriched encoding: reward motivation organizes cortical networks for hippocampal detection of unexpected events. Cereb Cortex 24:2160-2168. CrossRef Medline

Norman-Haignere SV, McCarthy G, Chun MM, Turk-Browne NB (2012) Category-selective background connectivity in ventral visual cortex. Cereb Cortex 22:391-402. CrossRef Medline

O'Carroll CM, Martin SJ, Sandin J, Frenguelli B, Morris RGM (2006) Dopaminergic modulation of the persistence of one-trial hippocampusdependent memory. Learn Mem 13:760-769. CrossRef Medline

Packard MG, White NM (1991) Dissociation of hippocampus and caudate nucleus memory systems by posttraining intracerebral injection of dopamine agonists. Behav Neurosci 105:295-306. CrossRef Medline

Peigneux P, Orban P, Balteau E, Degueldre C, Luxen A, Laureys S, Maquet P (2006) Offline persistence of memory-related cerebral activity during active wakefulness. PLoS Biol 4:e100. CrossRef Medline

Ranganath C, Yonelinas AP, Cohen MX, Dy CJ, Tom SM, D’Esposito M (2004) Dissociable correlates of recollection and familiarity within the medial temporal lobes. Neuropsychologia 42:2-13. CrossRef Medline

Shohamy D, Adcock RA (2010) Dopamine and adaptive memory. Trends Cogn Sci 14:464-472. CrossRef Medline

Shohamy D, Wagner AD (2008) Integrating memories in the human brain: hippocampal-midbrain encoding of overlapping events. Neuron 60:378389. CrossRef Medline

Staresina BP, Davachi L (2008) Selective and shared contributions of the hippocampus and perirhinal cortex to episodic item and associative encoding. J Cogn Neurosci 20:1478-1489. CrossRef Medline

Staresina BP, Alink A, Kriegeskorte N, Henson RN (2013) Awake reactivation predicts memory in humans. Proc Natl Acad Sci U S A 110:21159_ 21164. CrossRef Medline

Tambini A, Davachi L (2013) Persistence of hippocampal multivoxel patterns into postencoding rest is related to memory. Proc Natl Acad Sci U S A 110:19591-19596. CrossRef Medline

Tambini A, Ketz N, Davachi L (2010) Enhanced brain correlations during rest are related to memory for recent experiences. Neuron 65:280-290. CrossRef Medline

Uncapher MR, Otten LJ, Rugg MD (2006) Episodic encoding is more than the sum of its parts: an fMRI investigation of multifeatural contextual encoding. Neuron 52:547-556. CrossRef Medline

Wang SH, Redondo RL, Morris RGM (2010) Relevance of synaptic tagging and capture to the persistence of long-term potentiation and everyday spatial memory. Proc Natl Acad Sci U S A 107:19537-19542. CrossRef Medline

Wilson MA, McNaughton BL (1994) Reactivation of hippocampal ensemble memories during sleep. Science 265:676-679. CrossRef Medline

Wittmann BC, Schott BH, Guderian S, Frey JU, Heinze HJ, Düzel E (2005) Reward-related fMRI activation of dopaminergic midbrain is associated with enhanced hippocampus- dependent long-term memory formation. Neuron 45:459-467. CrossRef Medline 